\title{
Maternal erythrocyte ENT1-mediated AMPK activation counteracts placental hypoxia and supports fetal growth
}

\author{
Seisuke Sayama, ${ }^{1,2}$ Anren Song, ${ }^{1}$ Benjamin C. Brown, ${ }^{3}$ Jacob Couturier, ${ }^{4}$ Xiaoli Cai, ${ }^{1}$ Ping Xu, ${ }^{1}$ \\ Changhan Chen, ${ }^{1}$ Yangxi Zheng, ${ }^{1}$ Takayuki Iriyama, ${ }^{2}$ Baha Sibai, ${ }^{5}$ Monica Longo, ${ }^{5}$ \\ Rodney E. Kellems, ${ }^{1,6}$ Angelo D'Alessandro, ${ }^{3}$ and Yang Xia ${ }^{1,6}$ \\ 'Department of Biochemistry and Molecular Biology, The University of Texas Health Science Center at Houston, Houston, \\ Texas, USA. ${ }^{2}$ Department of Obstetrics \& Gynecology, University of Tokyo, Japan. ${ }^{3}$ Department of Biochemistry and \\ Molecular Genetics, University of Colorado School of Medicine, Aurora, Colorado, USA. ${ }^{4}$ Department of Internal Medicine, \\ ${ }^{5}$ Department of Obstetrics, Gynecology, and Reproductive Sciences, and ${ }^{6} \mathrm{Graduate}$ School of Biomedical Sciences, \\ University of Texas Health Science Center at Houston, Houston, Texas, USA.
}

Insufficient $\mathrm{O}_{2}$ supply is frequently associated with fetal growth restriction (FCR), a leading cause of perinatal mortality and morbidity. Although the erythrocyte is the most abundant and only cell type to deliver $\mathrm{O}_{2}$ in our body, its function and regulatory mechanism in FGR remain unknown. Here, we report that genetic ablation of mouse erythrocyte equilibrative nucleoside transporter 1 (eENT1) in dams, but not placentas or fetuses, results in FGR. Unbiased high-throughput metabolic profiling coupled with in vitro and in vivo flux analyses with isotopically labeled tracers led us to discover that maternal eENT1-dependent adenosine uptake is critical in activating AMPK by controlling the AMP/ATP ratio and its downstream target, bisphosphoglycerate mutase (BPGM); in turn, BPCM mediates 2,3-BPG production, which enhances $\mathrm{O}_{2}$ delivery to maintain placental oxygenation. Mechanistically and functionally, we revealed that genetic ablation of maternal eENT1 increases placental HIF-1 $\alpha$; preferentially reduces placental large neutral aa transporter 1 (LAT1) expression, activity, and aa supply; and induces FCR. Translationally, we revealed that elevated HIF-1 $\alpha$ directly reduces LAT1 gene expression in cultured human trophoblasts. We demonstrate the importance and molecular insight of maternal eENT1 in fetal growth and open up potentially new diagnostic and therapeutic possibilities for FGR.

Conflict of interest: The authors have declared that no conflict of interest exists.

Copyright: () 2020, American Society for Clinical Investigation.

Submitted: May 10, 2019

Accepted: April 15, 2020

Published: May 21, 2020

Reference information: /CI Insight. 2020;5(10):e130205.

https://doi.org/10.1172/jci.

insight.130205.

\section{Introduction}

Fetal growth restriction (FGR) is a condition in which a fetus fails to reach its full growth potential. The prevalence of FGR is known to be approximately $7 \%-15 \%$ of pregnancies, and it is the leading cause of perinatal mortality and morbidity, accounting for up to $75 \%$ of perinatal deaths $(1,2)$. FGR is also known to be associated with future development of metabolic syndrome in childhood and later adult life, referred to as fetal programming, and it has become a concern for the newborns in the long run $(3,4)$. However, we lack effective means to prevent or treat FGR, despite a variety of approaches undertaken (5). Because early detection and treatment of FGR will improve outcomes and substantially reduce morbidity and mortality from FGR (6), defining the molecular mechanisms underlying the disease is extremely important for the development of novel strategies for FGR prevention and treatment.

Normal placental development is essential for normal fetal growth. It has been long speculated that FGR is initially caused by abnormal placental development due to an insufficient supply of $\mathrm{O}_{2}$ and nutrients from mothers. As such, impaired placental development leads to FGR. Erythrocyte is the most abundant cell type in our body, acting as both a deliverer and sensor of $\mathrm{O}_{2}$. To function properly in $\mathrm{O}_{2}$ uptake, transport, and delivery, erythrocytes rely on sophisticated regulation of hemoglobin-oxygen $\left(\mathrm{Hb}-\mathrm{O}_{2}\right)$ affinity by endogenous allosteric modulators. One of the best-known allosteric modulators is 2,3-bisphosphoglycerate (2,3-BPG), a metabolic byproduct of glycolysis synthesized primarily in erythrocytes for the purpose of regulating $\mathrm{Hb}-\mathrm{O}_{2}$ affinity. It has been known for nearly 30 years that the concentration of 2,3-BPG in maternal erythrocytes is decreased significantly and its reduction is correlated with decreased capacity of $\mathrm{O}_{2}$ release from hemoglobin 

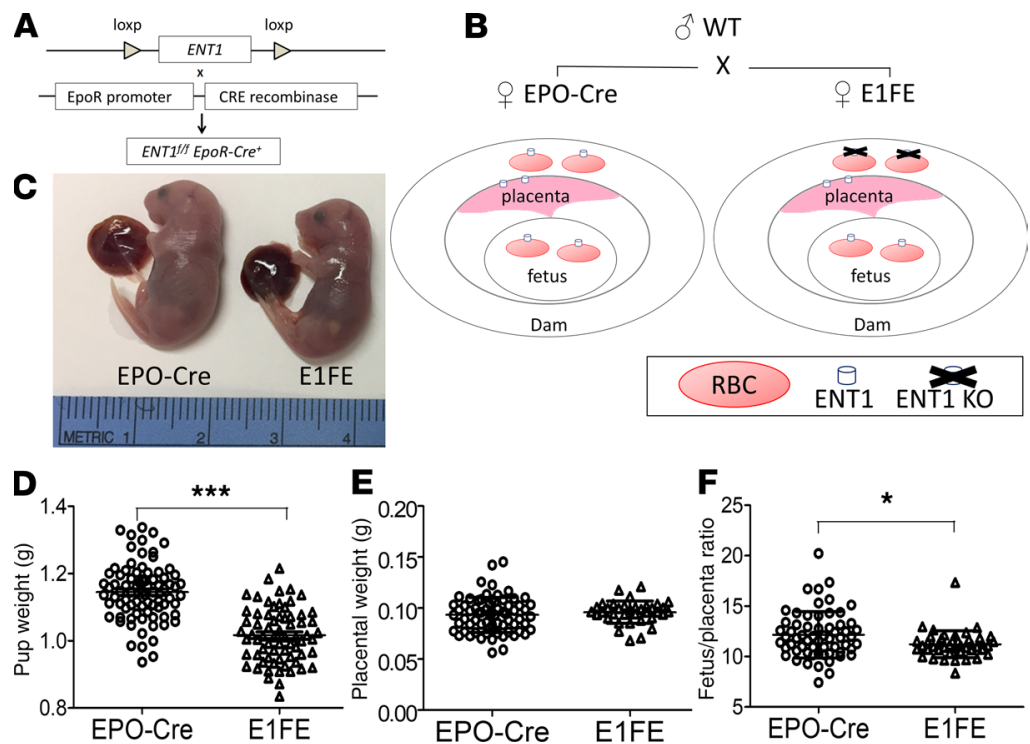

Figure 1. Ablation of ENT1 on maternal erythrocytes results in fetal growth restriction. (A) Description of lox-Cre system to generate erythrocyte-specific ENT1-KO mouse. (B) Mating strategy. E1FE female mouse was mated with WT male mouse, and EPO female was used as a control. (C) Representative picture of pups (E18.5) from both EPO and E1FE. (D-F) Graphs showing pup weight, placental weight, and fetus/placenta ratio. Values represent the mean \pm SEM. Number of dams used were $9-10 .{ }^{*} P<0.05,{ }^{* *} P<0.01$. Two-tailed Student's $t$ test was used for statistical analysis.

in pregnant women carrying babies with FGR compared with those delivering babies sized appropriate for gestational age $(\mathrm{AGA})(7,8)$. However, the molecular basis underlying reduction of maternal erythrocyte 2,3-BPG in FGR patients remains unknown, and the functional role of reduced maternal erythrocyte 2,3BPG in placental development and FGR is unclear. In prior work on metabolic adaptations to high-altitude hypoxia, we revealed the erythrocyte equilibrative nucleoside transporter 1 (eENT1) is the major transporter for quick uptake of extracellular adenosine and that eENT1 plays a key role to control extracellular adenosine under high-altitude acute hypoxia response (9). However, to our knowledge, the impact and molecular basis of eENT1 on adenosine metabolism and function during pregnancy remain unrecognized before this study.

Here, we report that a genetic ablation of eENT1 only in maternal erythrocytes is sufficient, to cause FGR. Unbiased high-throughput metabolic profiling coupled with in vitro flux analyses with stable isotopically labeled tracers revealed that genetic ablation of maternal eENT1 lowers intracellular adenosine during pregnancy and that adenosine taken up through eENT1 is largely converted to AMP in erythrocytes, resulting in decreased AMP/ATP ratio and AMPK activity, and thereby reduced BPG mutase (BPGM) activity, 2,3-BPG production, and $\mathrm{O}_{2}$ delivering capacity. These findings led us to further discover that eENT1 is a key purinergic component to control HIF-1 $\alpha$ expression and, in turn, preferentially regulates the expression and activity of large neutral aa transporter 1 (LAT1) expression and activity. Thus, among all of the metabolites detected, aa levels are the most reduced in the placentas, while their levels are the most accumulated in the plasma in mice with a genetic ablation of maternal eENT1. Finally, we conducted human translational studies demonstrating that increased HIF-1 $\alpha$ directly reduces LAT1 gene expression in cultured human trophoblast cell lines. We reveal that maternal eENT1 is a key molecule to maintain placental oxygenation, aa transporter expression and activity, and aa availability to support normal fetal growth by regulating AMPK/BPGM activity, 2,3-BPG production, and $\mathrm{O}_{2}$ delivery. These findings potentially add a chapter to FGR and pave a way to potentially novel diagnostic and therapy options for the disease.

\section{Results}

Genetic ablation of eENT1 in dams but not in placentas or fetus leads to FGR, independently of elevation of plasma adenosine and preeclampsia. Mature erythrocyte is the most abundant cell type in the blood, and its $\mathrm{O}_{2}$ delivery capacity is finely regulated by purinergic signaling components under hypoxia in both humans and mice at nonpregnant state under hypoxia (10). Notably, recent studies showed that erythrocytes predominantly express ENT1 and that ENT1 on the erythrocytes is responsible for adenosine uptake 

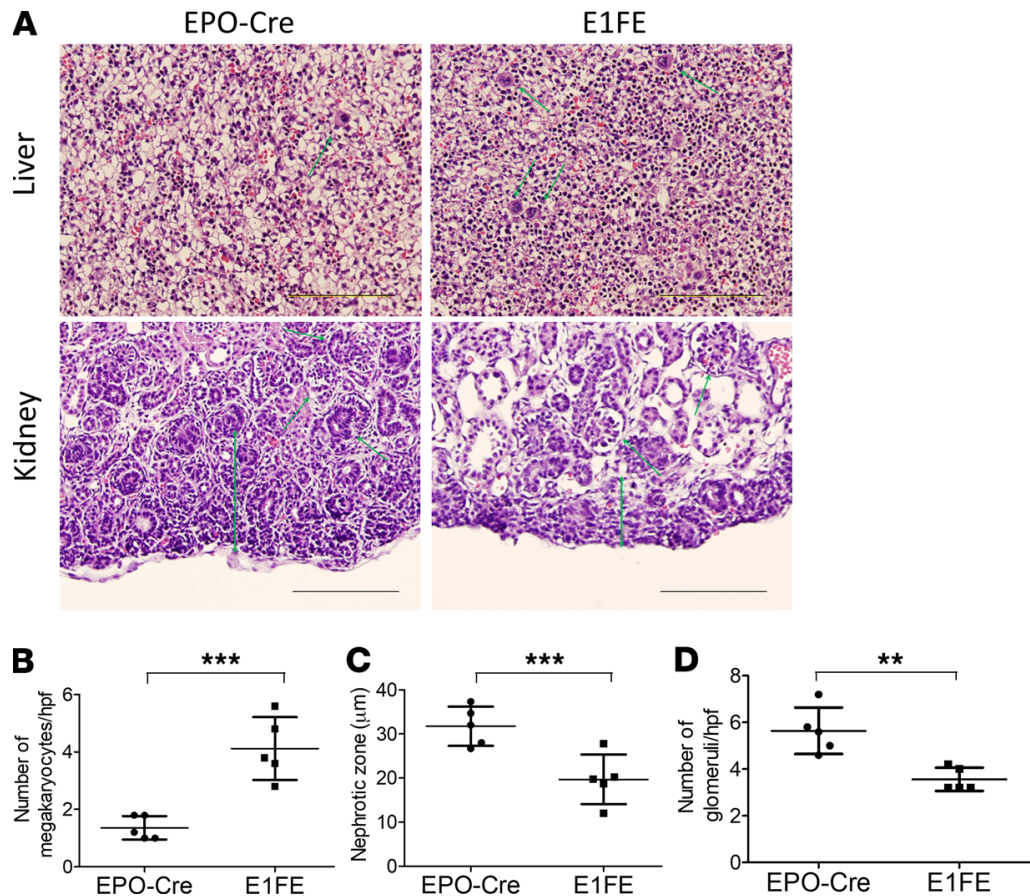

Figure 2. Maternal erythrocyte ablation of ENT1 results in multi-fetal organ restriction. (A) Fetal liver histology (H\&E staining) and fetal kidney histology (H\&E staining). Scale bars: $50 \mu \mathrm{m}$ (upper) and $25 \mu \mathrm{m}$ (lower). (B) Pups born from E1FE dams had substantially more megakaryocytes/hpf $(n=5)$. (C and D) Pups born from E1FE dams had significantly narrow nephrotic zone and less glomeruli/hpf $(n=5)$. Values represent the mean $\pm \mathrm{SEM} .{ }^{* *} P<0.01,{ }^{* *} P<0.001$. Twotailed Student's $t$ test was used for statistical analysis.

to eliminate accumulation of extracellular adenosine (9). However, the functional role of ENT1 on the erythrocytes in pregnancy remains unclear. Because maternal erythrocyte is the major cell type providing $\mathrm{O}_{2}$ to placentas and fetuses during pregnancy, we designed a sophisticated mating strategy to specifically delete ENT1 only on the maternal erythrocytes - not in the placentas or fetuses. Specifically, $E p o R-C r e^{+}(\mathrm{EPO})$ female mice were used as controls and Ent $t^{f / f}$-EpoR-Cre $(\mathrm{E} 1 \mathrm{FE})$ (Figure 1A) female mice were used as experimental mice and mated with WT male mice (Figure 1B). This mating strategy enabled us to focus on the effect of eENT1 during pregnancy, as the only difference between the control and the experimental group is the lack of ENT1 on the maternal erythrocytes. Western blot analysis validated that ENT1 was only deleted in erythrocytes but no other organs such as heart, lung, and brain (Supplemental Figure 2A; supplemental material available online with this article; https://doi. org/10.1172/jci.insight.130205DS1). As a result, the E1FE pregnancy showed FGR phenotype (1.017 $\pm 0.010 \mathrm{~g}$ ) with reduction of $12.9 \%$ in fetal weight compared with EPO group $(1.145 \pm 0.009 \mathrm{~g})$ (Figure $1, \mathrm{C}-\mathrm{E})$. The fetus/placenta ratio also showed $9.49 \%$ reduction (12.16 \pm 0.29 versus $11.21 \pm 0.20 \mathrm{~g})$, which is commonly seen in FGR mouse models (Figure 1F).

Next, we conducted histological studies of the fetal liver and kidney to define the fetal development. During normal mouse development, fetal liver is a major site for embryonic erythropoiesis. After E10, megakaryocytes start to decrease with advanced gestational age (11). H\&E staining showed significant increase of megakaryocytes in E1FE fetal liver compared with the controls ( $4.12 \pm 0.49$ versus $1.36 \pm 0.18$ / hpf) (Figure 2, A and B), suggesting a delay in fetal liver erythropoiesis in E1FE (12). To accurately define the erythroblast development in fetal liver, we conducted the flow cytometry analysis using 2 well-accepted erythroid surface markers, CD71 and Ter119 (13), and a hematopoietic stem cell progenitor marker, c-kit. We found that the populations of the early stages of erythroblast differentiation, including stage 1 (S1), S2, and S3, were significantly increased, whereas the population of S4, the later stage of erythroblast differentiation, was significantly decreased in E1FE fetal liver cells of E1FE compared with those of the controls (Figure 3, A and B). However, the population of fetal liver c-kit and CD71+ and CD71- cells did not differ between E1FE and the control (Supplemental Figure 5). Thus, flow cytometry analysis supports our conclusion that erythroid lineage maturation in the fetal liver cells of E1FE is restricted. 

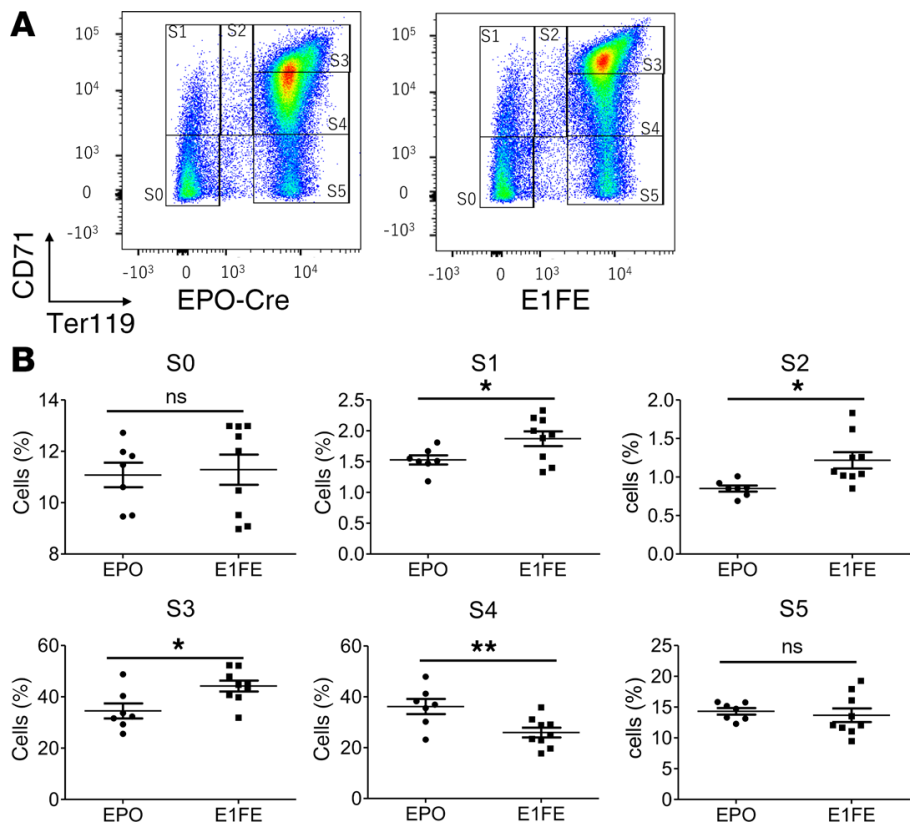

Figure 3. Expression of CD71 and Ter119 in fetal liver. (A) Flow cytometry of the fetal liver cells from E1FE and EPO-Cre dams. The erythroid progenitor cells were divided in 6 stages, SO-S5, according to the expression patterns of CD71 and Ter119. (B) Expression of CD71 is decreased in Ter119+ cells in the fetal liver derived from E1FE dams. Percentage of cells at S1, S2, and S3 was significantly increased in the E1FE group, whereas the percentage of cells at S4 stage was significantly decreased ( $n=7$ for EPO-Cre, 9 for E1FE) Values represent the mean $\pm \mathrm{SEM}$. ${ }^{*} P<0.05,{ }^{* *} P<0.01$. Two-tailed Student's $t$ test was used for statistical analysis.

The fetal kidneys also showed a decrease in nephrotic zone in E1FE fetal kidneys $(19.69 \pm 2.51 \mu \mathrm{m})$ compared with controls $(31.73 \pm 2.00 \mu \mathrm{m})$, as well as the number of glomeruli $(3.56 \pm 0.22$ versus $5.64 \pm$ 0.44/hpf, respectively), suggesting a renal developmental delay in E1FE fetuses (1) (Figure 2, A, C, and D). Thus, we concluded that genetic ablation of maternal eENT1 on E1FE dams leads to FGR.

Moreover, to determine if maternal ablation of ENT1 in the erythrocytes results in accumulation of plasma adenosine in E1FE dams, we measured adenosine levels in the maternal circulation in the controls and E1FE dams by HPLC. We found that maternal circulating adenosine levels showed no significant difference between controls and E1FE dams (Supplemental Figure 1A). FGR is frequently associated with preeclampsia (14). However, no hypertension or proteinuria were observed in E1FE dams (Supplemental Figure 1, B and C). Thus, we conclude that maternal ablation of eENT1 leads to FGR, independent of elevated plasma adenosine and preeclampsia.

Metabolomics profiling and isotopically labeled adenosine flux analysis reveal that uptake of extracellular adenosine by eENT1 is responsible for activation of $A M P K$ and BPGM and production of 2,3-BPG. In an effort to determine the molecular basis underlying the FGR phenotype seen in E1FE dams, we conducted a metabolomics screening of the erythrocytes isolated from controls and E1FE dams (Figure 4A). Metabolomics profiling identified 185 metabolites in the erythrocytes (Supplemental Table 1). Since ENT1 is the main transporter for the uptake of adenosine from outside of the erythrocytes (15), our metabolic profiling revealed that adenosine metabolism inside the erythrocytes is the most affected pathway when ENT1 on the membrane is lost on maternal erythrocytes. Specifically, the screening showed decrease in adenosine, AMP, and hypoxanthine, with reduction in adenine, ADP, and ATP levels (Figure 4B), while the changes of purinergic signaling components were not observed at nonpregnant state (Supplemental Figure 2F), implicating that ENT1-mediated uptake of adenosine is largely converted to AMP during pregnancy but not at nonpregnant state.

To test if adenosine transported by eENT1 is largely converted to AMP, we performed an in vitro flux experiment where we incubated either WT or ENT1-KO erythrocytes with isotopically ${ }^{13} \mathrm{C}^{15} \mathrm{~N}$-labeled adenosine and traced the metabolism of intracellular adenosine derived from the uptake of added extracellular adenosine (Figure 4D). Indeed, adenosine is rapidly phosphorylated to AMP upon uptake in order to promote energy metabolism and intracellular purinergic signaling. Intriguingly, ${ }^{13} \mathrm{C}^{15} \mathrm{~N}$-labeled AMP levels were lower in the ENT1-KO erythrocytes compared with the controls (Figure 4D). These findings provide 
A

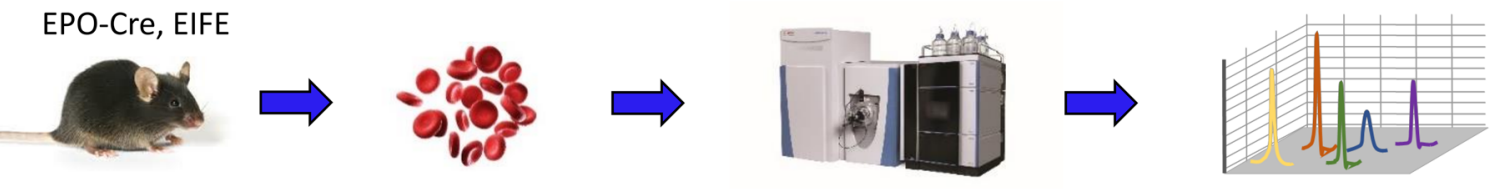

B
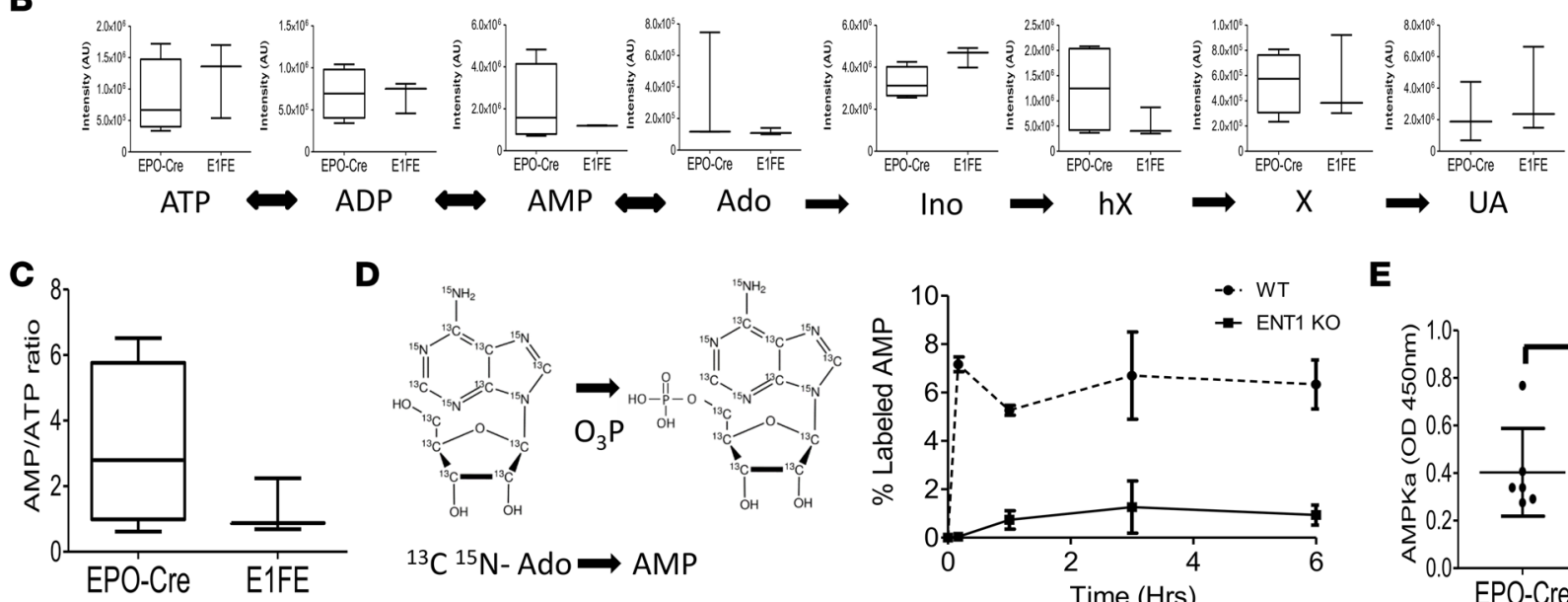

D

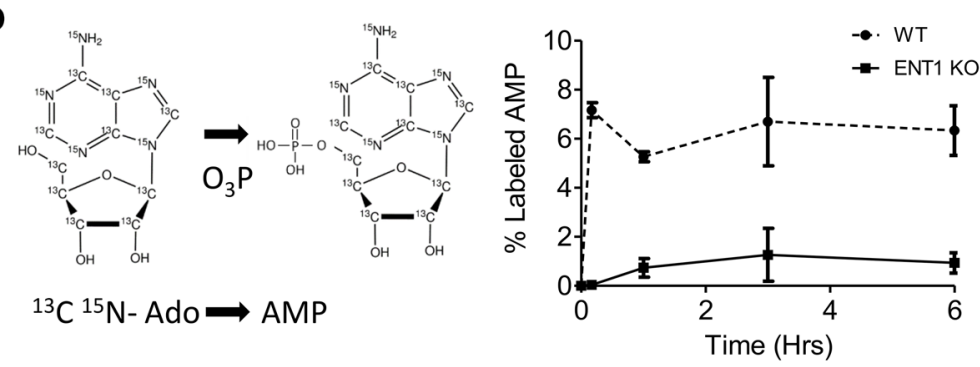

$\mathbf{E}$
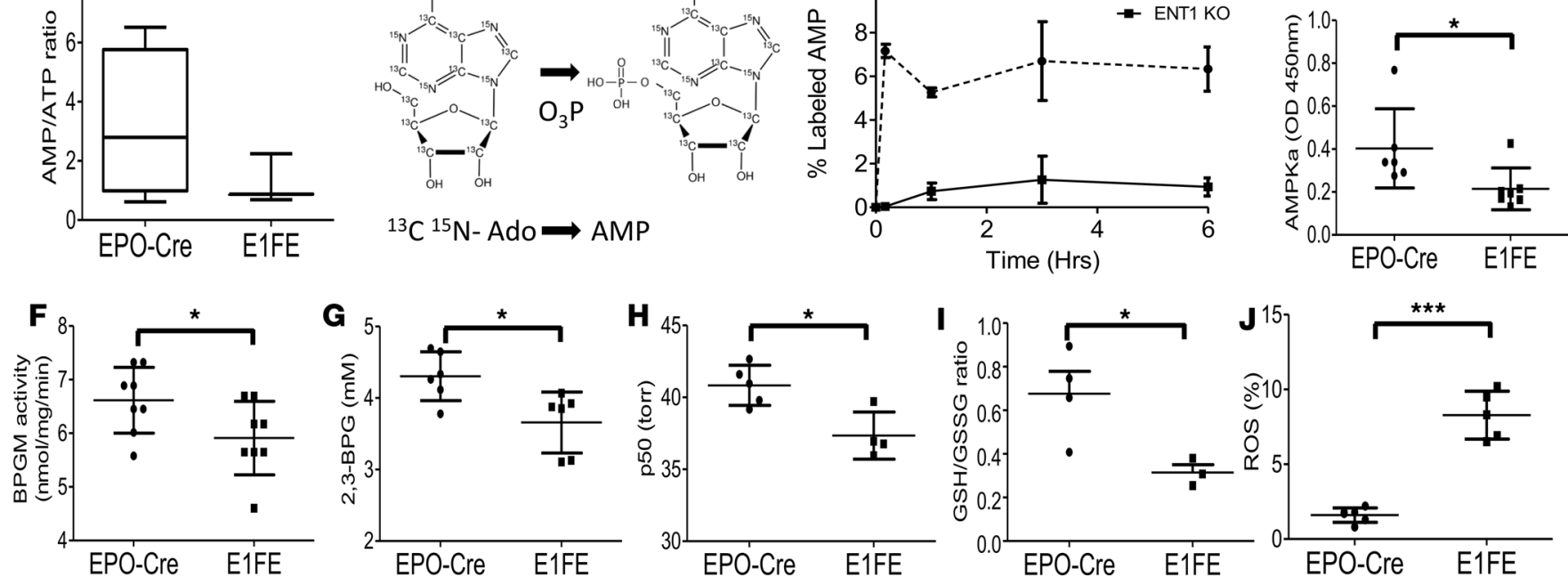

Figure 4. Metabolomic screening result from EPO and E1FE erythrocytes and ${ }^{13} \mathrm{C}$-labeled adenosine flux experiment with erythrocyte enzyme activity. (A) Description of metabolomic screening of the erythrocytes. (B) Metabolomics detected the changes of purine related metabolites in erythrocytes isolated from pregnant mice $(n=3)$. (C) AMP/ATP ratio was calculated from the metabolomic screening result $(n=3)$. (D) Description of ${ }^{13} \mathrm{C}$-labeled adenosine flux experiment. Result from flux experiment comparing erythrocytes from WT and ENT1-KO mice. AMP derived from the uptake of adenosine in the supernatant was reduced in erythrocytes from ENT1-KO mice $(n=3)$. (E-H) p-AMPK levels, BPGM activity, 2,3-BPG, and p50 levels were all decreased in erythrocytes from E1FE dams compared with control $(n=5-8)$. (I and J) Decreased GSH/GSSG ratio and accumulation of ROS in erythrocytes from E1FE dams compared with control. Values represent the mean $\pm \mathrm{SEM}$. ${ }^{*} P<0.05,{ }^{* * *} P<0.005(n=4-5)$. Twotailed Student's $t$ test was used for statistical analysis.

direct evidence that (a) the most affected metabolic pathway in the erythrocytes of E1FE dam is adenosine metabolism and (b) ENT1-mediated uptake of extracellular adenosine is largely converted to AMP but not ATP. As such, we observed reduced AMP/ATP ratio in erythrocytes of E1FE dams compared with the controls (Figure 4C), implicating that AMPK activity is likely reduced in maternal erythrocytes of E1FE dams compared with the controls.

Given the recent study showing that AMPK directly activates BPGM and leads to increased 2,3-BPG production in erythrocytes (16), we hypothesized that a decreased AMP/ATP ratio underlies the reduced 2,3-BPG production by lowering AMPK activity and subsequently decreasing BPGM activity. To test this hypothesis, we measured AMPK phosphorylation (p-AMPK) and BPGM activity in the erythrocytes in pregnant E1FE and EPO mice. As expected, we found both AMPK and BPGM activity were decreased in erythrocytes of E1FE dams compared with controls (Figure 4, E and F). Thus, we revealed that (a) adenosine derived from uptake of eENT1 is largely converted to AMP and (b) lack of maternal eENT1 lowers the AMP/ATP ratio and activity of AMPK and BPGM in maternal erythrocyte.

Moreover, we conducted complete blood count $(\mathrm{CBC})$ to determine if anemia occurs in E1FE before and during pregnancy. $\mathrm{CBC}$ studies showed that total $\mathrm{RBC}$ numbers and the hemoglobin levels did not differ between controls and E1FE before and during pregnancy (Supplemental Figure 2B). Thus, we conclude that eENT1 deletion in maternal erythrocytes does not cause pathological anemia but mainly affects 


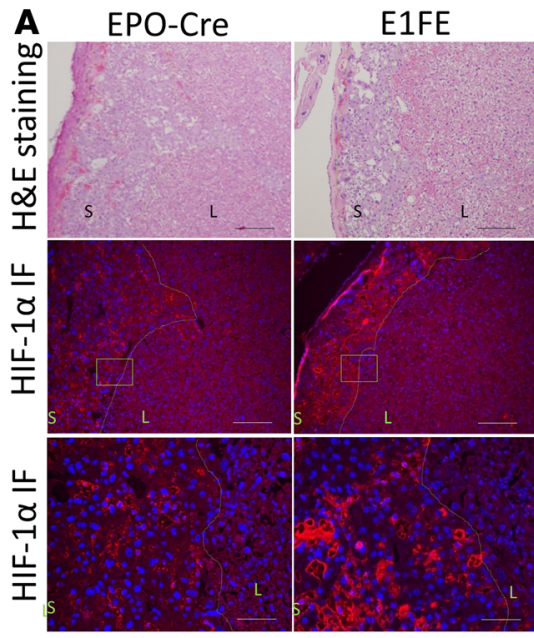

B
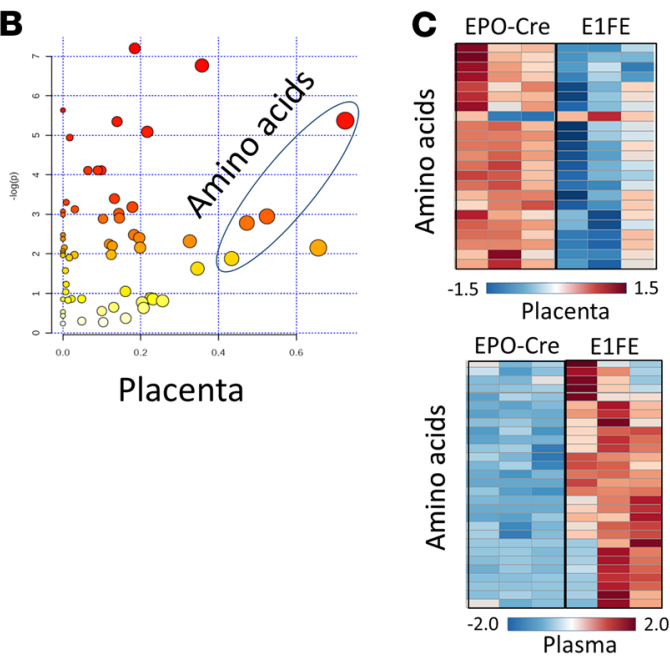

E

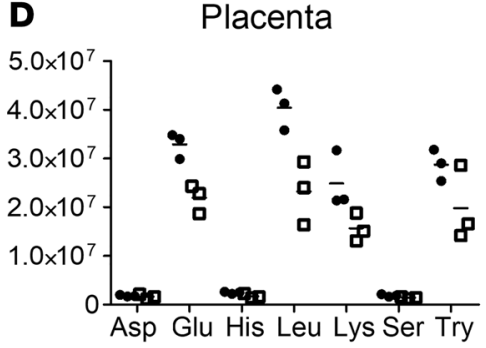

- EPO-Cre 口 $\mathrm{E} 1 \mathrm{FE}$
$5.0 \times 10^{7}$ $2.5 \times 10^{7}$ $5.0 \times 10^{0}$ $2.5 \times 10^{0}$

Figure 5. Placenta showing overexpression of HIF-1 $\alpha$ in E1FE dams and metabolomic screening result from EPO and E1FE placenta. (A) The placentas from E1FE show increase of HIF-1 $\alpha$ expression in the spongiotrophoblast zone. Scale bars: $200 \mu \mathrm{m}$ (top 2 rows) and $50 \mu \mathrm{m}$ (bottom row). L, labyrinth layer; S, spongiotrophoblast layer. (B) Pathways related to amino acid pathways are the most affected pathway in the E1FE placenta, compared with control. (C) Placentas from E1FE dams showed reduction in amino acids, whereas they were increased in plasma from E1FE dams compared with control. ( $\mathbf{D}$ and $\mathbf{E}$ ) Series of aa in the placenta were substantially reduced, whereas their levels were substantially elevated in maternal plasma. Values represent the mean \pm SEM.

adenosine uptake, in turn decreasing AMP levels and thus lowering AMPK and BPGM activity in mature erythrocytes of E1FE dams.

Genetic ablation of maternal eENT1 reduces 2,3-BPG levels and $\mathrm{O}_{2}$ delivery capacity in erythrocytes of E1FE dams carrying FGR fetuses. Early human studies showed that 2,3-BPG is reduced in maternal erythrocytes in pregnant women carrying FGR fetuses compared with the pregnant women carrying AGA fetuses, and its reduction is correlated with reduction in $\mathrm{O} 2$ tension at which hemoglobin is $50 \%$ saturated (p50) (7). However, the molecular basis and functional role of reduced 2,3-BPG and p50 from maternal erythrocytes in FGR remains undetermined. Our observation of FGR phenotype in E1FE pregnancy and our metabolomics profiling raises an intriguing possibility that eENT1-mediated uptake of extracellular adenosine is critical to maintain AMPK-BPGM activation and subsequent 2,3-BPG production during normal pregnancy. Consistent with human studies, we found that 2,3-BPG levels were significantly reduced in the maternal erythrocytes of E1FE dams compared with the controls (Figure 4G). Moreover, we measured p50, which is defined as the partial pressure of $\mathrm{O}_{2}$ to allow $50 \%$ of hemoglobin binding to $\mathrm{O}_{2}$. Specifically, the increased p50 indicates the increased $\mathrm{O}_{2}$ release capacity. As expected, we found that p50 was significantly reduced in maternal erythrocytes of E1FE dams compared with control mice (Figure $4 \mathrm{H}$ ). These findings revealed that genetic ablation of maternal eENT1 lowers 2,3-BPG levels and p50 in E1FE dams and results in FGR.

Lower p50 indicates higher affinity to $\mathrm{O}_{2}$ in erythrocytes, which may result in accumulation of ROS. Supporting this possibility, our metabolomics profiling revealed that erythrocyte GSH/GSSG ratio was reduced in E1FE dams compared with the controls (Figure 4I). Subsequently, we used flow cytometry to confirm the significant accumulation of ROS in erythrocytes of E1FE dams compared with the controls (Figure 4J). Thus, we demonstrated that uptake of adenosine by maternal eENT1 is critical to regulate $\mathrm{O}_{2}$ delivery capacity and oxidative stress by controlling AMPK and BPGM activity and, thus, 2,3-BPG production during pregnancy. 
A EPO-Cre E1FE

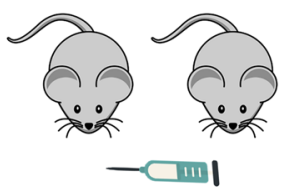

${ }^{13} \mathrm{C}^{15} \mathrm{~N}$ Amino Acid Mix (24h)

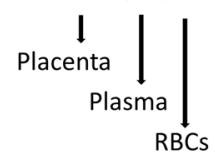

B<smiles>[13CH3][13CH2][13CH3]</smiles>

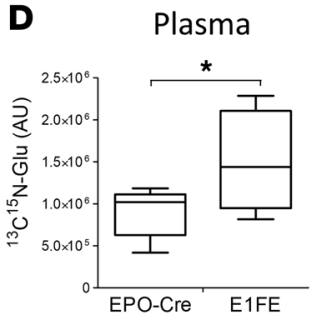

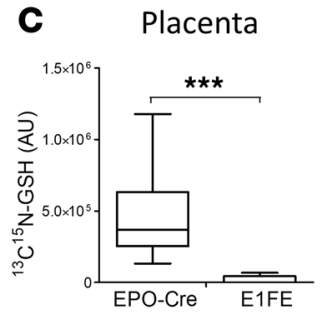

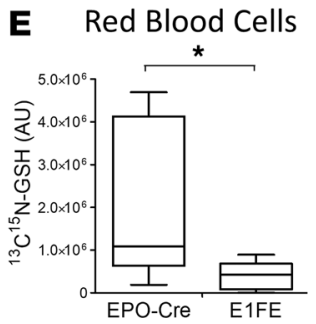

Figure 6. Decreased amino acid uptake by placenta leads to decreased CSH synthesis in both RBCs and placentas in E1FE pregnant mice. (A) In vivo flux analysis injecting isotopically labeled ${ }^{13} \mathrm{C}^{15} \mathrm{~N}$ amino acid mix in both controls and E1FE dams on E17.5, for 24 hours. (B) Glutamine is largely converted to glutamate and GSH in both erythrocytes and placentas. Thus, at the end of point, we compared the plasma ${ }^{13} \mathrm{C}^{15} \mathrm{~N}$ glutamate and placenta and RBCs CSH. (C) ${ }^{13} C^{15} \mathrm{~N}$-CSH was substantially decreased in the placentas in E1FE compared with the controls. (D) ${ }^{13} C^{15} \mathrm{~N}$-glutamate levels were accumulated in the plasma of E1FE compared with the controls. $(E)^{13} \mathrm{C}^{15} \mathrm{~N}-\mathrm{CSH}$ decreased in RBCs of E1FE mice, compared with control. Values represent the mean \pm SEM. ${ }^{*} P<0.05(n=5)$. Two-tailed Student's $t$ test was used for statistical analysis.

Placentas in E1FE dams suffer from insufficient oxygenation and a availability by downregulation of aa-specific transporters. Normal placental function is critical for normal fetal growth. Insufficient $\mathrm{O}_{2}$ supply from the erythrocytes of E1FE dams may induce hypoxia in the placentas. To address this hypothesis, we conducted immunofluorescence staining with HIF-1 $\alpha$ in the placenta. We found increased HIF-1 $\alpha$ expression in E1FE placenta, predominantly in the spongiotrophoblast layer (Figure 5A). The increased amount of HIF-1 $\alpha$ in the whole placenta from E1FE dams was also confirmed by Western blot analysis (Supplemental Figure 2, D and E). Furthermore, we demonstrated that no difference of hypoxia levels in multiple maternal tissues, including hearts and kidneys, between controls and E1FE dams (Supplemental Figures 3 and 4). Thus, we concluded that genetic ablation of maternal eENT1 induced placental hypoxia but no other maternal organs such as heart and kidney.

To further determine functional changes of hypoxic placentas in E1FE dams, we conducted metabolomics profiling in both the placenta and maternal plasma (Supplemental Tables 2 and 3). Intriguingly, among all of the metabolites altered, aa were the most reduced metabolites in E1FE placentas (Figure 5, B and C). In contrast, aa were the most accumulated in maternal plasma (Figure 5C). Especially, a series of aa in the placenta such as glutamate, aspartate, and leucine were substantially reduced, while their levels were substantially elevated in maternal circulation in E1FE compared with the control dams (Figure 5, D and E). These findings immediately suggest that decreased aa availability is likely due to reduced activity of aa transporters in E1FE placentas. To test this possibility, we conducted proof-of-principle in vivo flux analysis injecting isotopically labeled ${ }^{13} \mathrm{C}^{15} \mathrm{~N}$ aa mix in both controls and $\mathrm{E} 1 \mathrm{FE}$ dams on $\mathrm{E} 17.5$, for 24 hours (Figure 6A). Glutamine is largely converted to glutamate and GSH in both erythrocytes and placentas (Figure 6B). Thus, at the end point, we compared the plasma ${ }^{13} \mathrm{C}^{15} \mathrm{~N}$ glutamate and placenta and RBCs GSH. Consistent to our metabolomics, we found that ${ }^{13} \mathrm{C}^{15} \mathrm{~N}-\mathrm{GSH}$ is substantially decreased in the placentas in E1FE compared with the controls (Figure $6 \mathrm{C}$ ), while ${ }^{13} \mathrm{C}^{15} \mathrm{~N}$-glutamate levels were accumulated in the plasma of E1FE compared with the controls (Figure 6D), indicating reduced aa transporter activity in the placentas of E1FE. In addition, we noted that ${ }^{13} \mathrm{C}^{15} \mathrm{~N}-\mathrm{GSH}$ decreased in RBCs of E1FE mice (Figure 6E), consistent with increased oxidative stress in these RBCs, as measured in Figure 4J.

Finally, to determine which specific aa transporters are affected in the placentas in E1FE dams, we performed real-time PCR to quantify the mRNA of the known main transporters of aa in the mouse placenta. The real-time PCR showed reduction of LAT1 mRNA in E1FE placenta, where there was no difference in LAT2, SNAT1, or SNAT2 (Figure 7A). Western blot of the placenta lysates confirmed that the expression of LAT1 was indeed reduced (Figure 7B). 
A

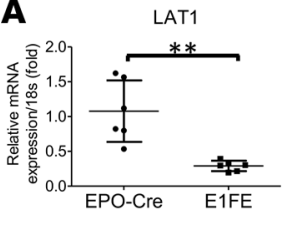

SNAT1

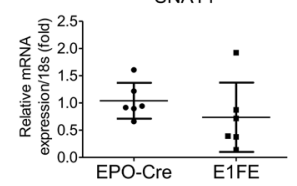

B

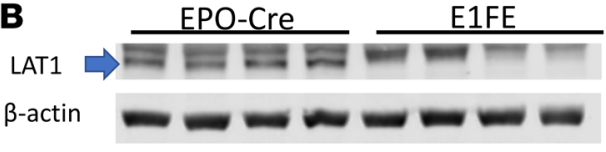

C
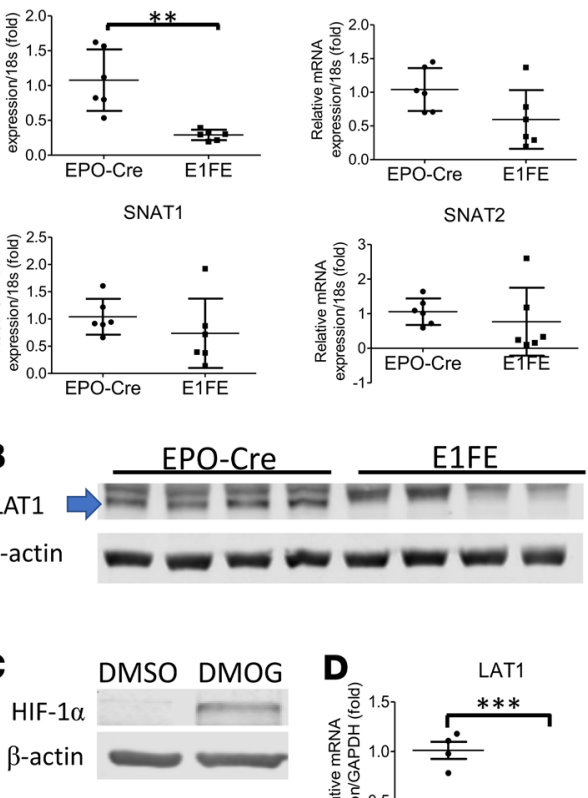

SNAT2

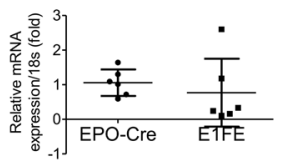

E1FE

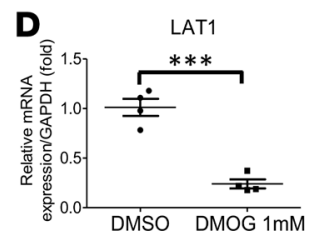

$\mathbf{E}$

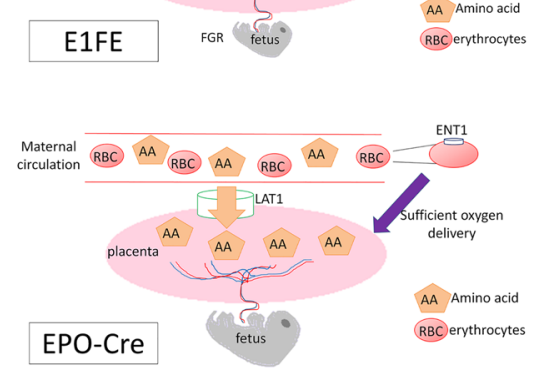

Figure 7. Expression of representative amino acid transporters in placentas from E1FE dams and controls. (A) mRNA of LAT1 was increased in placentas from E1FE dams, whereas there was no change in LAT2, SNAT1, and SNAT2. (B) Western blot results also show reduction in LAT1 expression in placentas from E1FE compared with control. (C) Western blot results show stabilized HIF-1 $\alpha$ with DMOG $1 \mathrm{mM}$ incubation. (D) mRNA of LAT1 were decreased in HTR cells with incubation of $1 \mathrm{mM}$ DMOG. Values represent the mean $\pm \mathrm{SEM}$. ${ }^{* *} P<0.01,{ }^{* * *} P<0.005$. (E) Working model. ENT1 plays an important role to regulate $\mathrm{O}_{2}$ release capacity and oxidative stress in maternal erythrocytes during pregnancy by controlling AMP/ATP ratio, AMPK-mediated BPGM activity, and 2,3-BPG production. ENT1-mediated $\mathrm{O}_{2}$ delivery from maternal erythrocytes controls placental oxygenation and aa transporter gene expression/activity to support normal fetal growth. Lacking maternal eENT1 leads to decreased $\mathrm{O}_{2}$ delivery to placenta, resulting in increased HIF-1 $\alpha$ and, decreased aa gene expression/activity, and fetal growth restriction. Two-tailed Student's $t$ test was used for statistical analysis.

HIF-1 $\alpha$-dependent downregulation of LAT1 and ENT1 in vitro human trophoblast cell lines. To validate our mouse finding and determine if HIF-1 $\alpha$ elevation directly downregulates LAT1 mRNA in humans, we treated a cultured human trophoblast cell line (HTR-8/SVneo cells) without or with DMOG, a cell permeable prolyl-4-hydroxylase inhibitor. First, we observed that DMOG upregulated HIF-1 $\alpha$ (Figure 7C). Next, we found that DMOG-mediated elevation of HIF-1 $\alpha$ increased LAT1 mRNA levels (Figure 7D). Thus, we conclude that elevated HIF-1 $\alpha$ underlies the reduction of LAT1 mRNA in cultured human trophoblasts.

\section{Discussion}

Despite the well-established role of hypoxia on FGR, little is known about the functional role of erythrocyte in FGR. Here, we reveal the mouse genetic evidence that maternal eENT1-dependent uptake of extracellular adenosine signaling via AMPK-mediated activation of BPGM and induction of 2,3-BPG, an erythroid specific negative allosteric modulator, plays a role in delivering adequate $\mathrm{O}_{2}$ to the placentas. Mouse genetic studies led us to further discover that insufficient $\mathrm{O}_{2}$ delivery from maternal erythrocytes deficient in ENT1 induces HIF-1 $\alpha$ expression but lowers expression of aa transporter (LAT1) in the placentas and, in turn, fails to transport sufficient aa from the mother to the fetus. Human translational studies showed that HIF-1 $\alpha$ stabilization directly downregulates LAT1 mRNA in cultured human trophoblasts. Overall, our findings reveal a beneficial role of intracellular erythrocyte adenosine functioning via the AMPK signaling cascade in promoting 2,3-BPG production and $\mathrm{O}_{2}$ delivery to placentas and, in turn, providing sufficient aa for fetal growth, thereby identifying potentially novel therapeutic possibilities for FGR (Figure 7E).

It has been known for nearly 3 decades that the maternal erythrocytes of pregnant women with FGR have increased $\mathrm{O}_{2}$ affinity due, in part, to the decrease in erythrocyte 2,3-BPG (7). As the only cell type delivering $\mathrm{O}_{2}$ to our body, little is known about the functional role of erythrocytes in FGR and the molecular 
mechanisms accounting for the downregulation of erythrocyte 2,3-BPG concentrations before our studies. Using a sophisticated genetic mating strategy, we found that genetic ablation of eENT1 in dams, but not in placentas or fetuses, results in FGR, indicating the importance of eENT1 for normal fetal growth. Recent studies showed that increased extracellular adenosine signaling via ADORA2B underlies the elevation of 2,3-BPG and induction of $\mathrm{O}_{2}$ release capacity via AMPK signaling in normal individuals facing high altitude and in sickle cell disease patients (10). However, deficiency in ENT1 only in maternal erythrocytes - not in placentas or fetuses - does not cause elevation of plasma adenosine. This finding rules out the possibility of elevated extracellular adenosine-mediated 2,3-BPG induction by activation of adenosine receptors (10). However, intracellular adenosine-regulating 2,3-BPG production remained unrecognized until our current studies, to our knowledge. Using high-throughput unbiased metabolomics profiling and isotopically labeled adenosine flux analysis to trace intracellular adenosine metabolism, we further discovered that intracellular adenosine taken up by maternal eENT1 is largely converted to AMP and that genetic disruption of maternal eENT1 reduced the AMP/ATP ratio, resulting in decreased activity of AMPK and BPGM and thus contributed to decreased 2,3-BPG production and $\mathrm{O}_{2}$ delivery capacity from maternal erythrocytes, as seen in humans with FGR. Thus, we have solved a 30 -year puzzle by providing mouse genetic evidence that erythrocyte intracellular adenosine taken up by ENT1 is required for normal fetal growth by maintaining a normal AMP/ATP ratio to activate AMPK and BPGM, generate 2,3-BPG, and release sufficient $\mathrm{O}_{2}$ during pregnancy. Furthermore, our metabolomic and in vivo aa flux analysis showed a decreased GSH/GSSG ratio and reduced GSH synthesis in maternal RBC of E1FE dams, indicating that ENT1 on maternal RBCs plays an important role to counteract oxidative stress by regulating GSH synthesis and/or GSSG recycling to GSH. Overall, we have revealed that ENT1 on maternal RBCs controls its $\mathrm{O}_{2}$ delivery by regulating 2,3-BPG production and counteracts oxidative stress by promoting GSH synthesis, as well as GSSG recycling to GSH.

FGR is associated with reduced fetal plasma concentration of multiple aa (17), despite normal or higher maternal concentration compared with normal pregnancies (18). Reduction in aa transporter activity is reported in the microvillous membranes of syncytiotrophoblasts obtained from placentas from FGR pregnancies $(19,20)$. LAT1 is an aa transporter also known as SLC7A5, which belongs to the APC (aa-polyamine-organocation) superfamily and forms a heterodimeric aa transporter interacting with the glycoprotein CD98 (SLC3A2). The complex is responsible for uptake of essential aa in multiple cancer cells, but it is only observed in the blood-brain barrier and placenta in normal tissues (21). The importance of LAT1 in embryonic development is shown with the use of LAT1-KO mice, which cannot go beyond midgestation stage (E11.5) (22). It has been reported that LAT1 expression is enhanced in cancer cells where HIF$1 \alpha$ is overexpressed (23). In contrast, in the placenta, LAT1 is known to be reduced under hypoxia (24). Although substantial research has focused on the role of insufficient placental oxygenation and aa availability in FGR, the molecular basis underlying hypoxic placentas and decreased placental aa transporters is poorly understood. Here, we demonstrate that decreased $\mathrm{O}_{2}$ delivery from maternal erythrocytes deficient in ENT1 leads to placental hypoxia. Notably, LAT1 is known to be a main transporter of leucine, and its concentration in the placenta was reduced by $33 \%$ but elevated in the maternal plasma compared with the controls in E1FE dams (Supplemental Figure 1, D and E), implicating that LAT1 transporter activity is downregulated. Supporting this finding, we further discovered that the expression of LAT1 levels were substantially reduced in the hypoxic placentas of E1FE dams. Finally, we provided human evidence that elevated HIF-1 $\alpha$ directly reduces gene expression of LAT1. Altogether, we revealed that reduced LAT1 mediated by elevated HIF- $1 \alpha$ due to decreased $\mathrm{O}_{2}$ delivery from maternal erythrocytes underlies decreased aa transporting from placentas for fetal growth. Early studies showed that LAT1 is expressed in human RBCs (25). Our in vivo flux experiments indicate that RBC uptake of aa is reduced, similar to the placentas in E1FE dams, implicating that LAT1 expression is reduced in maternal RBCs of E1FE dams. Whether LAT1 is reduced in maternal RBCs of E1FE dams, what the impact of reduced LAT1 is on maternal RBCs' function and metabolism, and how it is reduced are important points for future investigation.

In conclusion, we have solved a 30-year puzzle regarding how erythrocytes' $\mathrm{O}_{2}$ delivering capacity is associated with FGR. We demonstrated the function of erythrocytes and the metabolic basis of maintaining normal placental function to support adequate fetal growth during pregnancy. Both mouse genetic and human in vitro evidence supports a compelling working model that the intracellular adenosine taken up by maternal eENT1 regulates placental oxygenation by controlling 2,3-BPG production and $\mathrm{O}_{2}$ delivery capacity to the placentas via activation of AMPK-BPGM and, in turn, promotes adequate fetal growth by transporting sufficient aa from the mother to the placenta and the fetus (Figure 7E). Our findings of 
ENT1-mediated activation of AMPK-BPGM in regulating metabolism and $\mathrm{O}_{2}$ delivery capacity in maternal RBCs to control fetal growth implicate that variation of eENT1 function or acquired eENT1 dysfunction might occur in the mothers with FGR. Thus, our discovery of a ENT1-AMPK-BPGM signaling network in maternal RBCs is a potential candidate underlying downregulation of 2,3-BPG and $\mathrm{O}_{2}$ delivery in maternal RBCs that may cause FGR in humans. Our findings provide mechanistic insights into FGR and highlight the innovative therapeutic avenues.

\section{Methods}

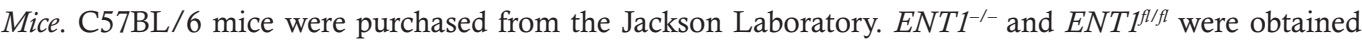
from Holger Eltzschig's lab at the University of Texas (McGovern Medical School). ENT1 ${ }^{f l / f l} E p o R C r e^{+}$mice were generated by cross-mating ENT1 flff with EpoRCre-GFP mice (26). Eight- to 12-week-old age-matched female mice were used for experimental mating with WT male mice aged 4-6 months. Four male mice were used, and 6 of both EPO-Cre and E1FE female mice were used for sample collection for the placenta and the maternal RBC and plasma. For the data collection for the fetal weight, 10 EPO-Cre and $9 \mathrm{E} 1 \mathrm{FE}$ female mice were used.

IHC and immunofluorescence in the placenta. Tissues were collected and fixed as described elsewhere on E18.5 (27). Tissue blocks were cut in 4- $\mu \mathrm{m}$ sections and stained with H\&E by standard procedures or were subject to immunofluorescence. Briefly, endogenous peroxidase activity was quenched by 10 minutes of incubation in a $3 \%$ hydrogen peroxide/methanol buffer. Antigen retrieval was done by incubating slides in sodium citrate buffer ( $\mathrm{pH} \mathrm{6.0)}$ at $89^{\circ} \mathrm{C}$ for 15 minutes. After blocking with the normal goat serum, the slides were incubated with antibody against HIF-1 $\alpha$ (1:100, Thermo Fisher Scientific, PA1-16601) and LAT1 (1:100, Santa Cruz Biotechnology Inc., sc-374232 [D-10]) in a humidified chamber at $4^{\circ} \mathrm{C}$ overnight. After the primary incubation, Alexa Fluor 594- or Alexa Fluor 488-conjugated donkey anti-rabbit IgG antibody (1:1000, Invitrogen; A-21207 and R37118, respectively) was used as a secondary antibody and counterstained with ProLong Gold Antifade Reagent with 4,6-diamidino-2-phenylindole (Cell Signaling Technology).

Histological analysis of the fetal organs. The number of glomeruli in the fetal kidney samples was assessed blindly by counting and averaging the number of glomeruli in 10 random high-power microscopic fields per sample.

CD71, Ter119, and c-kit quantification with flow cytometry in fetal liver. Tissues were collected as described above on E18.5. Fetal liver preparation and staining for flow cytometry was described before (28). In brief, fetal liver was dissected from the fetus, and the tissue was passed through 40- $\mu \mathrm{m}$ nylon mesh and rinsed with HBSS buffer (Thermo Fisher Scientific) to prepare for single cell solution. Cell staining of CD71 and Ter119 conjugated with PE-Cy7 and Pacific Blue (BioLegend), respectively, and CD71 and c-kit conjugated with PE-Cy7 and Pacific Blue (BioLegend), respectively, were placed on ice for 1 hour. Then, the cells were spun down and analyzed by using BD LSTII (BD Biosciences), and data were processed using FlowJo software (TreeStar Inc.).

Hypoxy probe staining and quantification in maternal heart and kidney. For the purpose of detection of tissue hypoxia, hypoxia probe (50 mg/Kg body weight, Hypoxyprobe) was injected through i.p. injection 60 minutes before the animal was sacrificed on E18.5. Before tissue collection, animals were perfused with 30 $\mathrm{mL}$ cold PBS through left ventricle injection, and the tissues were fixed as described above. After antigen retrieval, the slides were incubated with hypoxy probe antibody (1:100, Hypoxyprobe) in a humidified chamber at $4^{\circ} \mathrm{C}$ overnight. After the primary incubation, Alexa Fluor 488-conjugated donkey anti-rabbit IgG antibody (1:1000, Invitrogen, R37118) was used as a secondary antibody and counterstained with ProLong Gold Antifade Reagent with 4,6-diamidino-2-phenylindole (Cell Signaling Technology). Signal intensity was analyzed using ImageJ $(\mathrm{NIH})$. One slide/animal $(n=3,2-3$ images/slide, more than 5 locations) were used for intensity measuring/slide.

Blood collection and preparation from mice. A total of $1 \mathrm{~mL}$ of blood was collected in a tube with EDTA anticoagulant containing $1 \mu \mathrm{L}$ of $10 \mathrm{mmol} / \mathrm{L}$ dipyridamole (Sigma-Aldrich, D9766) and $1 \mu \mathrm{L}$ of $10 \mathrm{mmol} / \mathrm{L}$ ADA inhibitor deoxycoformycin (DCF, TOCRIS Bioscience, 2033), and the sample was centrifuged at 2,400 $g$ for 5 minutes to isolate plasma, buffy coat, and erythrocytes.

ELISA measurement of erythrocyte phosphorylation of AMPKa at Thr172. A total of $10 \mu \mathrm{L}$ of frozen erythrocytes was lysed with RIPA buffer, and the protein concentration was measured with a commercially available kit (Thermo Fisher Scientific, Pierce BCA Protein Assay Kit, 23227). Phosphorylation levels of erythrocyte AMPK $\alpha$ at Thr172 were quantitatively measured by using a commercially available ELISA kit (Cell Signaling Technology, PathScan Phospho-AMPKa [Thr172] Sandwich ELISA Kit, 7959), and the value was normalized with protein concentration of the lysate. 
Measurement of erythrocyte 2,3-BPGM activity. Frozen erythrocyte pellets were used for protein extraction following the protocol as mentioned above. Erythrocyte protein extract was subsequently used for measurement of 2,3-BPGM activity as described previously, with modification (29). Briefly, erythrocyte protein extract was incubated in $100 \mu \mathrm{L}$ prepared reaction mixture $(100 \mathrm{mM}$ Triethanolamine $\mathrm{pH} 7.6,1 \mathrm{mM}$ MgSO4, 4 mM ATP, 3 mM 3-phosphoglycerate, 10 unit phosphoglycerate kinase; Sigma-Aldrich, 97634) for 20 minutes at $30^{\circ} \mathrm{C}$. Then, the reaction was terminated by adding $25 \mu \mathrm{L}$ of Trichloroacetic acid and subsequently centrifuged at $10,000 \mathrm{~g}$ for 5 minutes. A total of $100 \mu \mathrm{L}$ of the supernatant was mixed with $17 \mu \mathrm{L}$ of $1.8 \mathrm{M}$ Tris base, and the 2,3-BPG level of the mixture was measured by using a commercial assay (Roche, 2,3DPG assay kit,10148334001) as previously described (30).

2,3-BPG analysis and erythrocyte $\mathrm{O}_{2}$ release capacity ( $\left.p 50\right)$ measurement. Erythrocyte 2,3-BPG was isolated as indicated before and quantified by a commercially available kit (Roche) (30). For p50 measurement, $10 \mu \mathrm{L}$ of whole blood aliquot were mixed with $4.5 \mathrm{~mL}$ Hemox Buffer (TCS Scientific Corporation), $10 \mu \mathrm{L}$ anti-foaming reagent (TCS Scientific Corporation), and $20 \mu \mathrm{L} \mathrm{22 \%} \mathrm{BSA} \mathrm{in} \mathrm{PBS.} \mathrm{Then,} \mathrm{the} \mathrm{mixture} \mathrm{was} \mathrm{injected} \mathrm{in} \mathrm{the}$ Hemox Analyzer (TCS Scientific Corporation) for measurement of an $\mathrm{O}_{2}$ equilibrium curve at $37^{\circ} \mathrm{C}$.

Metabolomics profiling. Erythrocytes $(100 \mu \mathrm{L})$ and plasma samples $(100 \mu \mathrm{L})$ were isolated as described above, and placentas were isolated and snap frozen in liquid nitrogen. The samples were stored at $-80^{\circ} \mathrm{C}$ before metabolomics analyses. RBCs, RBC supernatants, plasma, and placenta were extracted (in a $1: 10,1: 25,1: 25$, and $15 \mathrm{mg} / \mathrm{mL}$ dilution, respectively) with a mixture of ice-cold methanol, acetonitrile, and water (5:3:2 v/v). Extractions were performed by vigorous vortexing for 30 minutes at $4^{\circ} \mathrm{C}$; then, insoluble materials were pelleted by centrifugation for 10 minutes at $18,213 \mathrm{~g}$ at $4^{\circ} \mathrm{C}$. Clarified extraction supernatants were analyzed by ultra-high-pressure liquid chromatography coupled online to mass spectrometry (UHPLC-MS) using a Thermo Vanquish UHPLC coupled to a Thermo Q Exactive MS. Samples were randomized and run in positive and negative ion modes (separate runs) at an injection volume of 10 or $20 \mu \mathrm{L}$, respectively (RBCs/placenta and RBC supernatants/plasma, respectively). UHPLC phases were water (A) and acetonitrile (B) supplemented with $0.1 \%$ formic acid for positive mode runs and $1 \mathrm{mM}$ ammonium acetate for negative mode runs. Metabolites were separated on a Kinetex C18 column $(2.1 \times 150 \mathrm{~mm}, 1.7 \mu \mathrm{m}$, Phenomenex $)$ using a 5-minute gradient method with the following conditions: flow rate, $0.45 \mathrm{~mL} / \mathrm{min}$; column temperature, $45^{\circ} \mathrm{C}$; sample compartment temperature, $7^{\circ} \mathrm{C}$; solvent gradient, $0-0.5$ minutes at $5 \% \mathrm{~B}, 0.5-1.1$ minutes at $5 \%-95 \% \mathrm{~B}, 1.1-2.75$ minutes hold at $95 \% \mathrm{~B}, 2.75-3$ minutes at $95 \%-5 \% \mathrm{~B}, 3-5$ minutes hold at $5 \% \mathrm{~B}$. The mass spectrometer was operated in full MS mode at a resolution of 70,000, scan range 65-900 $\mathrm{m} / \mathrm{z}$, maximum injection time $200 \mathrm{~ms}$, microscans 2, automatic gain control (AGC) $3 \times 106$ ions, electrospray source voltage 4.0 $\mathrm{kV}$, capillary temperature $320^{\circ} \mathrm{C}$, and sheath gas 45 , auxiliary gas 15 , and sweep gas 0 (all nitrogen). Raw data files were converted to mzXML format using MassMatrix (Case Western Reserve University, Cleveland, Ohio, USA) and analyzed using Maven (Princeton University, Princeton, New Jersey, USA). Quantification is relative based on integrated peak areas of extracted ion chromatograms at the MS1 level. Instrument stability and quality control was assessed using replicate injections of a technical mixture every 10 runs as described $(31,32)$.

Adenosine flux experiment in vitro. Erythrocytes for culture were isolated from blood collected with heparin as an anticoagulant. Packed erythrocytes were purified using Percoll density purification (MilliporeSigma) as previously described (33). Packed erythrocytes from WT and ENT1 KO mice were washed 3 times with culture media (F-10 nutrients mix; Invitrogen) and suspended to $4 \%$ hematocrit. A total of $1 \mathrm{~mL}$ of erythrocytes was added to each well of a 12 -well plate and cultured with $5 \mu \mathrm{M}$ of ${ }^{13} \mathrm{C}_{10}{ }^{15} \mathrm{~N}_{5}$-labeled adenosine (MilliporeSigma) either under hypoxia $\left(1 \% \mathrm{O}_{2}\right)$ or normoxia. The flux was analyzed by looking for the monoisotopic masses of labeled and endogenous metabolites associated with adenosine pathways in the software Maven (Princeton); this was then prepared for graphical display using GraphPad Prism 6.

In vivo tracing experiments with stable isotope-labeled aa into mice. Pregnant mice were injected to retro orbital sinus with ${ }^{13} \mathrm{C}^{15} \mathrm{~N}$-labeled aa mixture (MilliporeSigma) $(4 \mathrm{~mL} / \mathrm{kg}$ BW of $10 \times$ diluted aa mixture) on E17.5, 24 hours before sacrifice. Placenta, RBCs, and plasma were extracted from the mice and frozen at $-80^{\circ} \mathrm{C}$; they were then shipped to University of Colorado Denver (Denver, Colorado, USA) for metabolic flux analysis. The mice samples were extracted in the same manner as the metabolomics profiling. The flux analysis was performed by determining isotopologues of labeled aa and metabolites downstream, namely +7.0128 glutamate and glutathione in the software Maven (Princeton); this was then prepared for graphical display using GraphPad Prism 6 software. 
Human trophoblast cell culture. HTR-8/SVneo cells, part of an immortalized human trophoblast cell line (34), were plated at $2 \times 10^{6}$ cells per well in a 10 -cm Petri dish overnight (Thermo Fisher Scientific). The next day, serum-free RPMI 1640 (Thermo Fisher Scientific) media was used and cells were incubated with either DMSO or $1 \mathrm{mM}$ of DMOG (Tocris) for 24 hours. Then, the cells were either lysed for RNA extraction or protein extraction for further experiments.

Quantitative real-time PCR. mRNA was isolated and converted to cDNA with a commercially available kit. (QIAGEN) Syber green was used for the analysis of all genes measured using the following primers: Mouse LAT1, forward 5'-CATCATCGGCTCTGGCATCTTCGTG-3' and reverse 5'-TCTGCTGCAGGTTGGACGCATCAC-3'; mouse LAT2, forward 5'-GCCCTCACCTTCTCCAACTA-3' and reverse 5'-CCATGTGAGGAGCAACAAAC-3'; mouse SNAT1, forward 5'-TTACCAACCATCGCCTTC-3' and reverse 5'-ATGAGAATGTCGCCTGTG-3'; SNAT2, forward 5'-GGTATCTGAACGGTGACTATCTG-3' and reverse 5'-TCTGCGGTGCTATTGAATGC-3'; mouse 18s, forward 5'-GTAACCCGTTGAACCCCATT-3' and reverse 5'-CCATCCAATCGGTAGTAGCG-3'; human HIF-1A, forward 5'-TGCTCATCAGTTGCCACTTC-3' and reverse 5'-TCCTCACACGCAAATAGCTG-3'; human GAPDH, forward 5'-TGCACCACCAACTGCTTAGC-3' and reverse 5'-ACAGTCTTCTGGGTCGCAGTG-3'.

Western blot. Placentas stored in $-80^{\circ} \mathrm{C}$ was homogenized in RIPA buffer (Thermo Fisher Scientific) in the presence of Halt proteinase and phosphatase inhibitor (Thermo Fisher Scientific) to yield total lysate. A total of $50 \mu \mathrm{g}$ of the total placenta lysates was loaded for Western blot detection of membrane-bound LAT1 with an antibody against LAT1 (1:1000, Santa Cruz Biotechnology Inc., sc-374232 [D-10]) and then probed with secondary antibodies labeled with IRDye fluorophores (LI-COR). The antibody/antigen complexes were scanned and detected using the ODYSSEY infrared imaging system and software (LI-COR).

Growth pattern. Mating pair was set as C57BL/6 male mates with EPO-Cre or E1FE female; pups were weighed at days 21, 28, 35, 42, and 49 (Supplemental Figure 2C). Six pups per mating pair were used for the study. Statistical analysis was performed using 2-way ANOVA.

Statistics. All data are expressed as means \pm SEM. For growth pattern weight studies, statistical analysis was performed using 2-way ANOVA. Data were analyzed for statistical significance using Prism 5 software (GraphPad Software, Inc.). Two-tailed Student's $t$ tests (paired or unpaired as appropriate) were applied in a 2-group analysis. $P<0.05$ was considered significant.

Study approval. All animal protocols were in accordance with the University of Texas, Medical School at Houston guidelines (protocol no. 17-0056).

\section{Author contributions}

SS and YX designed the research studies. SS conducted all the experiments except for flow cytometry, metabolomic screening, and flux analyses. PX and YZ helped in mating mice. AS provided nonpregnant metabolomic screening data, Western blot for eENT1 specific deletion and LAT1 expression in the placenta, fetal liver erythropoiesis characterization, and immuno-staining of heart and kidney with hypoxy probe. CC monitored growth curve. JC and XC conducted flow cytometry. BCB and AD performed metabolomic screening and flux analyses. SS, BCB, AD, and YX analyzed the data. AS and TI assisted in some of the experiment design. SS, ML, BS, REK, and YX wrote the manuscript.

\section{Acknowledgments}

This work is supported by NIH HL137990 and HL136969 (to YX), American Heart Association grant 19CDA34670038 (to AS), and Webb-Waring Early Career Award by the Boettcher Foundation (to AD).

Address correspondence to: Yang Xia, Department of Biochemistry and Molecular Biology, University of Texas McGovern Medical School at Houston, 6431 Fannin Street, Houston, Texas 77030, USA. Phone: 713.500.5039; Email: yang.xia@uth.tmc.edu.

1. Alexander GR, Kogan M, Bader D, Carlo W, Allen M, Mor J. US birth weight/gestational age-specific neonatal mortality: 1995-1997 rates for whites, hispanics, and blacks. Pediatrics. 2003;111(1):e61-e66.

2. Trudell AS, Cahill AG, Tuuli MG, Macones GA, Odibo AO. Risk of stillbirth after 37 weeks in pregnancies complicated by small-for-gestational-age fetuses. Am J Obstet Gynecol. 2013;208(5):376.e1-376.e7.

3. de Rooij SR, Painter RC, Holleman F, Bossuyt PM, Roseboom TJ. The metabolic syndrome in adults prenatally exposed to the Dutch famine. Am J Clin Nutr. 2007;86(4):1219-1224. 
4. Veenendaal MV, et al. Transgenerational effects of prenatal exposure to the 1944-45 Dutch famine. BJOG. 2013;120(5):548-553.

5. Groom KM, David AL. The role of aspirin, heparin, and other interventions in the prevention and treatment of fetal growth restriction. Am J Obstet Gynecol. 2018;218(2S):S829-S840.

6. Alfirevic Z, Neilson JP. Doppler ultrasonography in high-risk pregnancies: systematic review with meta-analysis. Am J Obstet Gynecol. 1995;172(5):1379-1387.

7. Brown EG, et al. The relationship of maternal erythrocyte oxygen transport parameters to intrauterine growth retardation. Am J Obstet Gynecol. 1990;162(1):223-229.

8. Lackman F, Capewell V, Gagnon R, Richardson B. Fetal umbilical cord oxygen values and birth to placental weight ratio in relation to size at birth. Am J Obstet Gynecol. 2001;185(3):674-682.

9. Song A, et al. Erythrocytes retain hypoxic adenosine response for faster acclimatization upon re-ascent. Nat Commun. 2017;8:14108.

10. Liu H, et al. Beneficial Role of Erythrocyte Adenosine A2B Receptor-Mediated AMP-Activated Protein Kinase Activation in High-Altitude Hypoxia. Circulation. 2016;134(5):405-421.

11. Matsumura G, Sasaki K. Megakaryocytes in the yolk sac, liver and bone marrow of the mouse: a cytometrical analysis by semithin light microscopy. J Anat. 1989;167:181-187.

12. Irani RA, et al. The detrimental role of angiotensin receptor agonistic autoantibodies in intrauterine growth restriction seen in preeclampsia. J Exp Med. 2009;206(12):2809-2822.

13. Socolovsky M, Nam H, Fleming MD, Haase VH, Brugnara C, Lodish HF. Ineffective erythropoiesis in Stat5a(-/-)5b(-/-) mice due to decreased survival of early erythroblasts. Blood. 2001;98(12):3261-3273.

14. Kaufmann P, Black S, Huppertz B. Endovascular trophoblast invasion: implications for the pathogenesis of intrauterine growth retardation and preeclampsia. Biol Reprod. 2003;69(1):1-7.

15. Song A, et al. Erythrocytes retain hypoxic adenosine response for faster acclimatization upon re-ascent. Nat Commun. $2017 ; 8: 14108$.

16. Liu H, et al. Beneficial Role of Erythrocyte Adenosine A2B Receptor-Mediated AMP-Activated Protein Kinase Activation in High-Altitude Hypoxia. Circulation. 2016;134(5):405-421.

17. Cetin I, et al. Umbilical amino acid concentrations in normal and growth-retarded fetuses sampled in utero by cordocentesis. Am J Obstet Gynecol. 1990;162(1):253-261.

18. Cetin I, et al. Maternal concentrations and fetal-maternal concentration differences of plasma amino acids in normal and intrauterine growth-restricted pregnancies. Am J Obstet Gynecol. 1996;174(5):1575-1583.

19. Glazier JD, et al. Association between the activity of the system A amino acid transporter in the microvillous plasma membrane of the human placenta and severity of fetal compromise in intrauterine growth restriction. Pediatr Res. 1997;42(4):514-519.

20. Mahendran D, Donnai P, Glazier JD, D'Souza SW, Boyd RD, Sibley CP. Amino acid (system A) transporter activity in microvillous membrane vesicles from the placentas of appropriate and small for gestational age babies. Pediatr Res. 1993;34(5):661-665.

21. Scalise M, Galluccio M, Console L, Pochini L, Indiveri C. The Human SLC7A5 (LAT1): The Intriguing Histidine/Large Neutral Amino Acid Transporter and Its Relevance to Human Health. Front Chem. 2018;6:243

22. Ohgaki R, et al. Essential Roles of L-Type Amino Acid Transporter 1 in Syncytiotrophoblast Development by Presenting Fusogenic 4F2hc. Mol Cell Biol. 2017;37(11):e00427-16.

23. Fuchs BC, Bode BP. Amino acid transporters ASCT2 and LAT1 in cancer: partners in crime? Semin Cancer Biol. 2005;15(4):254-266.

24. Nelson DM, et al. Hypoxia reduces expression and function of system A amino acid transporters in cultured term human trophoblasts. Am J Physiol, Cell Physiol. 2003;284(2):C310-C315.

25. Dosier LBM, Premkumar VJ, Zhu H, Akosman I, Wempe MF, McMahon TJ. Antagonists of the system L neutral amino acid transporter (LAT) promote endothelial adhesivity of human red blood cells. Thromb Haemost. 2017;117(7):1402-1411.

26. Heinrich AC, Pelanda R, Klingmüller U. A mouse model for visualization and conditional mutations in the erythroid lineage. Blood. 2004;104(3):659-666.

27. Iriyama T, et al. Elevated placental adenosine signaling contributes to the pathogenesis of preeclampsia. Circulation. 2015;131(8):730-741.

28. Morrison SJ, Hemmati HD, Wandycz AM, Weissman IL. The purification and characterization of fetal liver hematopoietic stem cells. Proc Natl Acad Sci USA. 1995;92(22):10302-10306

29. Gallego C, Graña X, Carreras J. Increase of 2,3-bisphosphoglycerate synthase/phosphatase during maturation of reticulocytes with high 2,3-bisphosphoglycerate content. Mol Cell Biochem. 1991;102(2):183-188.

30. Zhang Y, et al. Detrimental effects of adenosine signaling in sickle cell disease. Nat Med. 2011;17(1):79-86.

31. Nemkov T, D'Alessandro A, Hansen KC. Three-minute method for amino acid analysis by UHPLC and high-resolution quadrupole orbitrap mass spectrometry. Amino Acids. 2015;47(11):2345-2357.

32. Nemkov T, Hansen KC, D'Alessandro A. A three-minute method for high-throughput quantitative metabolomics and quantitative tracing experiments of central carbon and nitrogen pathways. Rapid Commun Mass Spectrom. 2017;31(8):663-673.

33. Pertoft H, Laurent TC, Låås T, Kågedal L. Density gradients prepared from colloidal silica particles coated by polyvinylpyrrolidone (Percoll). Anal Biochem. 1978;88(1):271-282.

34. Graham CH, et al. Establishment and characterization of first trimester human trophoblast cells with extended lifespan. Exp Cell Res. 1993;206(2):204-211. 